\title{
A GENERALIZATION OF MOORE'S THEOREM ON SIMPLE TRIODS
}

\author{
GAIL S. YOUNG, JR.
}

R. L. Moore has proved that the plane does not contain uncountably many mutually exclusive simple triods. ${ }^{1}$ The generalization to spaces of higher dimension given below appears to have escaped notice, and to be of some interest.

DEFINITION. If $n$ is a non-negative integer, by a $T_{n}$-set we shall mean a continuum which is the sum of an $n$-cell, $g$, and an arc, $t$, such that $g \cdot t$ is a point which is an end point of $t$ and a relatively interior point of $g$. The point $g \cdot t$ will be called a junction point.

Obviously, a $T_{1}$-set is a simple triod.

Theorem. Euclidean n-space does not contain uncountably many mutually exclusive $T_{n-1}$-sets.

Proof. Supose that the theorem is false. Then for some positive number $\epsilon$ there exists an uncountable collection, $G$, of mutually exclusive $T_{n-1}$-sets such that the junction point of each is at distance greater than $\epsilon$ from the boundary of its $(n-1)$-cell. There is a point, $P$, which is a point of condensation of the set of all junction points of elements of $G$; let $U$ be a spherical domain of $n$-space with center $P$ and radius less than $\epsilon / 2$. If the point $X$ of $U$ is a junction point of an element $T$ of $G$, let $g(X)$ denote the component that contains $X$ of the intersection of $U$ and the $(n-1)$-cell of $T$. It is an easy consequence of the Alexander duality theorem that $g(X)$ separates $U$. Hence the collection, $G^{\prime}$, of all sets $g(X)$ is an uncountable collection of cuttings of $U$, and it is clearly non-separated. By a theorem due to Whyburn, ${ }^{2} G^{\prime}$ contains an uncountable saturated subcollection, $G^{\prime \prime}$. But if $g(X)$ is an element of $G^{\prime \prime}, U$ contains an arc, $t$, which is in the $T_{n-1}$-set of $G$ that contains $X$ and which has only $X$ in common with $g(X)$. Since the elements of $G$ are mutually exclusive, no element of $G^{\prime \prime}$ separates a point of $t$ from $g(X)$ in $U$, which is a contradiction.

\section{Purdue University}

Presented to the Society, April 29, 1944; received by the editors March 25, 1944.

${ }^{1}$ In fact, Moore has proved that the plane does not contain uncountably many mutually exclusive triodic continua. See Concerning triods in the plane and the junction points of plane continua, Proc. Nat. Acad. Sci. U.S.A. vol. 14 (1928) pp. 85-88, and Concerning triodic continua in the plane, Fund. Math. vol. 13 (1929) pp. 261-263.

${ }^{2}$ Theorem 2.2 of chap. 3 of his book, Analytic topology, Amer. Math. Soc. Colloquium Publications, vol. 28. 